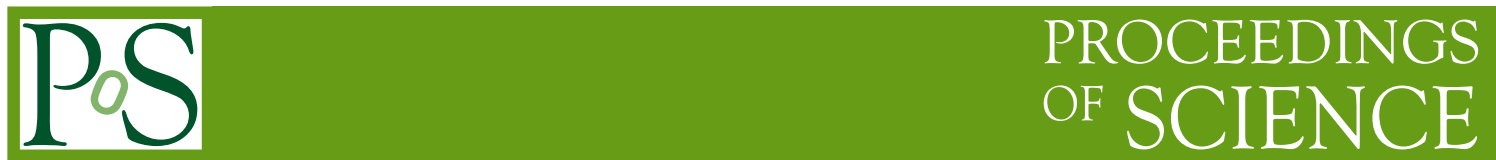

\title{
Direct Dark Matter and Axion Detection with CUORE
}

\author{
CECILIA G. MAIANO* \\ on the behalf of CUORE collaboration \\ UNIVERSITA 'DEGLI STUDI DI MILANO BICOCCA, INFN MILANO BICOCCA, ITALY \\ E-mail: cecilia.maiano@mib.infn.it
}

\begin{abstract}
CUORE will consist of $988 \mathrm{TeO}_{2}$ bolometers of $750 \mathrm{~g}$ each and it is expected to reach such a low background level which allows a sensitivity to $\beta \beta 0 v$ down to the inverted hierarchy of neutrino masses: it is a powerfull tool to test the neutrino nature and absolute scale mass. Given the high mass, the good energy resolution, and the low background the CUORE experiment can search for rare processes such as Dark Matter interactions (they are sensitive to nuclear recoils), provided the energy thresholds are low enough. CUORE, thanks to its large mass, could potentially look for an annual modulation of the counting rate at low energies. A trigger and a pulse shape identification algorithm had been developed which allow to lower the energy threshold down to the few $\mathrm{keV}$ region. We present the preliminary results obtained on an array made of four CUORE-like crystals, and the prospects for a dark matter search in CUORE. Finally a brief discussion on the potential axion study with CUORE detector will be presented
\end{abstract}

VIII International Workshop on the Dark Side of the Universe

June 10-15, 2012

Búzios, Rio de Janeiro, Brasil

\footnotetext{
${ }^{*}$ Speaker.
} 


\section{Bolometers and the CUORE experiment}

The CUORE experiment is designed mainly to search for the neutrinoless double beta decay of ${ }^{130} \mathrm{Te}$ [1] . The observation of this decay would determine theMajorana nature of these particles, unlike all the other fermions which are Dirac particles, and would set a absolute scale for the neutrino mass. The existing CUORE energy threshold, achieved using a trigger algorithm applied to the raw data samples, is of the order of tens of $\mathrm{keV}$. CUORE could also search for dark matter (DM) interactions, provided that the energy thresh- old and the radioactive background are sufficiently low. DM candidates such as weak interacting massive particles (WIMPs) and axion-like particles (ALPs). Electronics spikes, mechanical vibrations, and temperature fluctuations can produce pulses that, if not properly identified, generate nonphysical background. The lower the energy released in the bolometer, the more difficult it is to discriminate between physical and nonphysical pulses.

\section{Experimental setup}

The algorithms developed were tested on an array made of four CUORE-like crystals bolometers operated by the CUORE collaboration at the Gran Sasso underground laboratory (LNGS) in Italy. The main purpose of the test was to check (form the radioactive and detector's performances point of view) one of the first production batches of CUORE crystals. Briefly, a CUORE bolometer is composed of two main parts, a $\mathrm{TeO}_{2}$ crystal and a neutron transmutation doped Germanium (NTD-Ge) thermistor.When energy is released in the crystal, its temperature increases and changes the thermistor's resistance. The thermistor is biased with a constant current, and the voltage across it constitutes the signal.

\section{Data anlysis and results}

The trigger and the pulse shape identification algorithms operate on data samples processed using the matched filter [2]. This filter is used to estimate the amplitude of a signal, and can be used when the expected shape of the signal and the noise power spectrum of the detector are known [3]. Data are divided into slices and are then filtered in the frequency domain using the optimum filter (OF) algorithm, maximizing the signal to noise ratio. The trigger is implemented as a simple threshold trigger on the filtered samples and the threshold is set a priori in terms of the OF theoretical resolution. To remove non-physical pulses we fit the filtered pulses with the known shape of physical pulses, the $\chi^{2} \mathrm{f}$ is used as shape parameter. To measure the detection efficiency, an energy scan with the external heater was performed using sequences of pulses from the keV region up to $\sim 100 \mathrm{keV}$. The measured efficiency increases with energy and reaches a plateau. In the first bolometer the plateau efficiency amounts to $90 \%$ and is reached at $\sim 2.5 \mathrm{keV}$; The residual $10-20 \%$ inefficiency in the plateau is due to the trigger dead time, which is mainly due to ${ }^{210} \mathrm{Po}$ decays. The four bolometers array took 19 days of data. We estimated the CUORE sensitivity toWIMPs assuming that the background differential rate will be equal to the measured value in the four bolometers array, and that all bolometers will have a $3 \mathrm{keV}$ threshold. The sensitivity to a WIMP modulation signal in CUORE-0 (39 $\mathrm{kg}$ of $\mathrm{TeO}_{2}, 3 \mathrm{y}$ ) and in CUORE (741 kg of $\mathrm{TeO}_{2}, 5 \mathrm{y}$ ) is evaluated with toy MonteCarlo's. CUORE-0 and CUORE will not be as sensitive as experiments able 
Figure 1: CUORE-5y (in red) and CUORE0-3y (in black) sensitivities compared with the actual limits on WIMP-nucleon elastic scattering cross section. DAMA/LIBRA $3 \sigma$ evidence with ion channeling in dark red and without ion channeling in pink, Edelweiss II in green, XENON100 in blue, CDMS in magenta.

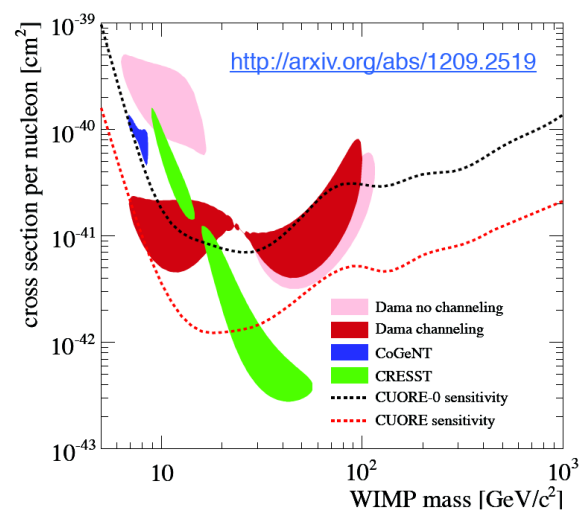

Figure 2: Exclusion plots for one and two sigma conincidence levels for fa as a function of the nuclear $\mathrm{S}$ parameter values.

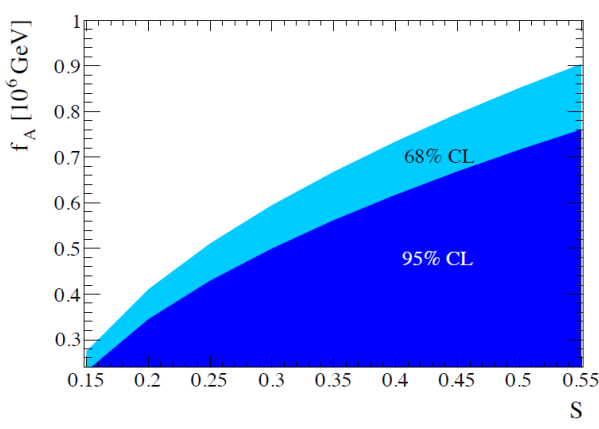

to discriminate nuclear recoils. CUORE, however, could investigate the same parameter space of the DAMA/LIBRA experiment and could be the only experiment other than DAMA/LIBRA looking for an annual modulation of dark matter interactions (fig.1). Finally the axion was postulated to solve the strong CP problem in QCD: the theory predicts the existence of a neutral spin-zero pseudoscalar particle, called axion, with a non-zero mass ma. In $\mathrm{TeO}_{2}$ crystals the only available possibility is a search for hadronic axions which could be emitted from the Sun inM1 transitions, between the first $14.4 \mathrm{keV}$ thermally excited and the ground state in ${ }^{57} \mathrm{Fe}$, and absorbed in the $\mathrm{TeO}_{2}$ detector by axioelectric effect. An analysis on a CUORE test detector is ongoing (fig.2).

\section{References}

[1] R. Ardito et. al., CUORE: A cryogenic underground observatory for rare events, arXiv:hep-ex/0501010 (2005) [hep-ex/0501010].

[2] E. Gatti and P.F. Manfredi, Processing the signals from solid state detectors in elementary particle physics, Riv. Nuovo Cim. 9N1 (1986) 1

[3] S. Di Domizio, F. Orio and M. Vignati, Lowering the energy threshold of large-mass bolometric detectors, IOP P UBLISHING FOR SISSA. 